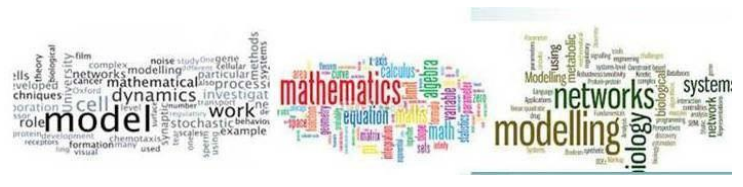

Journal, Advancesin Mathematical \& computational Sciences

Vol. 8 No. 3 September, 2020

www.mathematics-computationaljournal.info

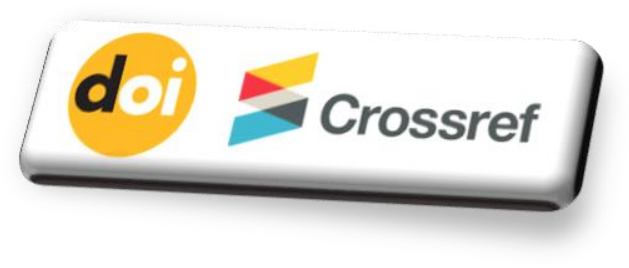

Journal of Advances in Mathematical \& Computational Sciences An International Pan-African Multidisciplinary Journal of the SMART Research Group International Centre for IT \& Development (ICITD) Southern University Baton Rouge, LA, USA

(c) Creative Research Publishers - Available online at https://www.isteams.net/socialinformaticsjournal DOI: dx.doi.org/10.22624/AIMS/MATHS/ V8N3P4

CrossREF Member Listing - https://www.crossref.org/06members/50go-live.html

\title{
Signal with Blake2, Chacha20 and Poly1305
}

\author{
${ }^{1}$ Atiku, A.U. \& ${ }^{2}$ Sajoh, D.I. \\ 1,2Department of Computer Science \\ Modibbo Adama University of Technology \\ Yola, Adamawa State, Nigeria \\ 1ahmed.atiku@mautech.edu.ng, 2disajoh@mautech.edu.ng
}

\begin{abstract}
Messaging applications are now adopting the concept of end to end encryption. Signal uses the Signal Protocol which has no known vulnerabilities for its end to end encryption. The protocol was explained in this work and possibilities of improvements were explored. It was discovered that the protocol implementation uses the SHA-512 hash, AES in CBC mode for encryption and HMAC for authentication. These Cryptographic Primitives were replaced with more efficient alternatives, Blake2, Chacha20 and Poly1305 respectively. An application was created to test the running time of the proposed cryptographic primitives against the existing one. From the results, Blake2b performs better than SHA-512 when hashing keys, short text, and 1.4 MB file. Similarly, Chacha20/Poly1305 performs better than AES/CBC/HMACsha256 in both encrypting and decrypting short text as well as 1.4 MB file. The proposed changes were implemented in the Signal Application. Automatic test in the Signal application was used to ensure that changes made to the code did not cause any logic errors. To further test the application, it was installed on another phone and messages were sent. The security code was also verified.
\end{abstract}

Keywords: Signal, Blake2, Chacha20, Poly1305

Atiku, A.U. \& Sajoh, D.I..(2020): Signal with Blake2, Chacha20 and Poly1305. Journal of Advances in Mathematical \& Computational Sc. Vol.8, No. 3. Pp 3748. DOI: dx.doi.org/10.22624/AIMS/MATHS/ V8N3P4. Available online at www.isteams.net/mathematics-computationaljournal.

\section{INTRODUCTION}

Signal is a messaging application developed by Open Whisper Systems. The application has end to end encryption that is built on a protocol called Signal Protocol, which was also developed by Open Whisper Systems[2]. The protocol is a combination of two protocols: Textsecure, which uses the concept of double ratchetting to ensure future secrecy and Redphone[8][10]. Signal protocol didn't get much attention in the research world until its integration to WhatsApp in 2015 [4]. [8] did a formal security analysis of the protocol focusing on the multistage Authenticated Key Exchange (AKE) protocol. They showed that the protocol is resistant to Unknown Keyshare Attacks and did not find any serious vulnerabilities. 


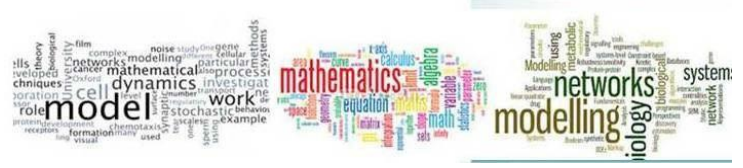

Journal, Advancesin Mathematical \& computational Sciences

Vol. 8 No. 3 September, 2020

www.mathematics-computationaljournal.info

Considering WhatsApp's large user base, a comparison of the protocol implementation was done in [10]. They identified the differences in the implementation and justified WhatsApp's decision to sacrifice security for usability in some parts of its implementation. The protocol is also used to provide end to end encryption in Facebook, Wire and Google Allo[15].

These applications, including WhatsApp and Signal, are used mostly in mobile devices which have limited resources. It is therefore important that the Signal Protocol is efficient. The focus of this paper is improving the efficiency of the protocol implementation in Signal using faster encryption algorithms without compromising security.

\section{THE SIGNAL PROTOCOL}

\section{Asymmetric Keys Used}

- ika, IKA: Alice's Identity key pair

- eka, EKA: Alice's Ephemeral key pair

- rckai, RCKAi: Alice's ith Ratchet Key pair

- ikb, IKB: Bob's Identity key pair

- spkb, SPKB: A pair of Bob's Signed Pre-key

- $(o p k b, O P K B)^{*}$ : multiple pairs of Bob's one time pre-keys. A single one will be written without the*

- $r c k b^{i}, R C K B^{i}$ : Bob's ith Ratchet Key

The keys written in lower case represent the private keys while those in upper case are the public keys.

\section{Symmetric Keys used}

- rki: The ith Root Key

- cki: The ith Chain Key

- $m k i$ : The ith Message Key

\section{Other Abbreviations}

- ECDH: Elliptic Curve Deffie Hellman key exchange

- $\mathrm{DH}(\mathrm{ka}, \mathrm{KB})$ : An ECDH using a private key belonging to Alice and the other Bob's public key

- $\operatorname{Sig}_{k}(M)$ : Signing a message M with a private key

- $\operatorname{Ver}_{K B}($ Sign): Verify Bob's Signature with his public key

- AD: Associated Data

- AEAD: Authenticated Encryption with Associated Data. This is a set-up that provides both encryption and MAC of a message with its $A D$

- $E_{k}(M, A D)$ : Encrypting a message $M$ along with its $A D$ using a key $k$ in an $A E A D$ set-up

- $D_{k}(C, A D)$ : Decrypting a message $C$ along with its $A D$ using a key $k$ in an $A E A D$ set-up

- $K D F(s e c)$ : A key derivation function that takes in a secret sec and uses it to derive two new secrets

- KDF2(sec1, sec2): A key derivation function that takes in a two secrets sec1, sec2 and uses it to derive two new secrets

The Signal Protocol description can be divided into three stages: Key Registration, Trust Establishment and Messaging. 


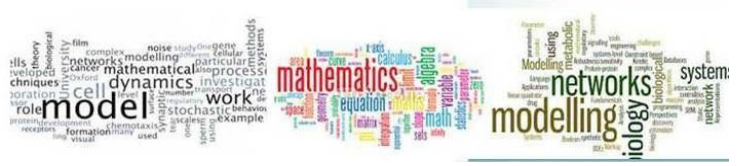

Journal, Advancesin Mathematical \& computational Sciences

Vol. 8 No. 3 September, 2020

www.mathematics-computationaljournal.info

\subsection{Key Registration}

At install time, Bob most first register his phone number which will be used as his identity. To confirm that he is the actual owner of that number, the server sends an SMS (Short Message Service) with a confirmation code to the phone number entered. Bob now enters this confirmation code in the Application. If verified, the phone number will be registered to the server and Bob will generate some elliptic curve key pairs and send the public keys to the server as shown in Fig.1. Once the server registers Bob's details.

Other users can communicate with Bob using the protocol. The use of confirmation code is not specified in the Signal Protocol, but it is used at implementation stage by the messaging application. The Signal Protocol is based on a Trust On First Use (TOFU) scheme and it does not provide any cryptographic guarantee that an attacker cannot send his own elliptic curve keys to the server with Bob's identity[13]. However, the protocol provides a means of authentication with a security code generated from the public keys as explained in section 2.2. The keys sent to the server by Bob are IKB, SPKB and OPKB*. IKB is a permanent key and it only changes when Bob reinstalls the application or changes his device. SPKB is periodically changed and sent to the server together with the signature SignB. When the server is running out of OPKB, it notifies Bob and he sends more OPKB*.

Bob Server Bob's Phone

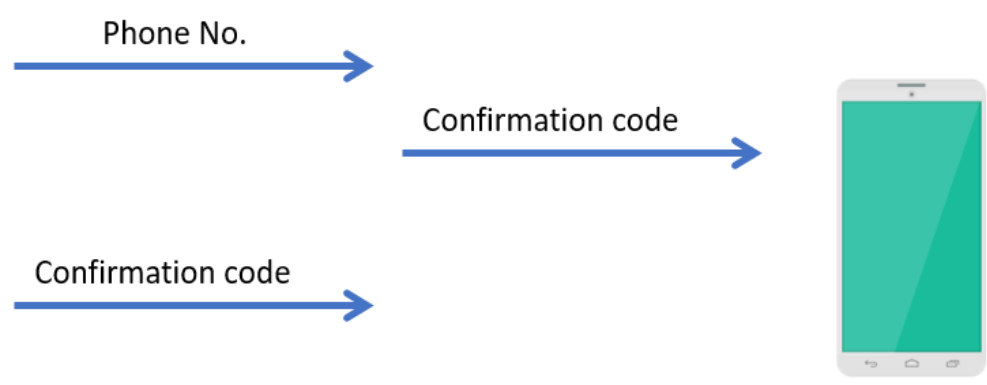

ikb, IKB

spkb, SPKB

$\left.\operatorname{SignB}=\operatorname{Sig}_{i k b}(S P K B)\right)$

(opkb, OPKB)*

IKB, SPKB, SignB, OPKB*

Add record:

IKB,

SPKB,

SignB

OPKB*,

Phone No.

Figure 1: The Registration Process 


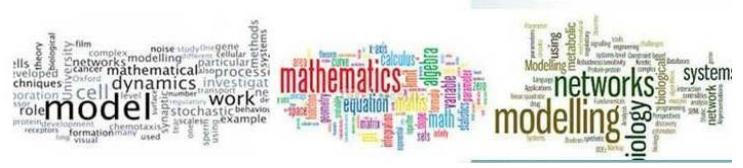

Journal, Advancesin Mathematical \& computational Sciences

Vol. 8 No. 3 September, 2020

www.mathematics-computationaljournal.info

\subsection{Key Exchange and Trust Establishment}

If Alice tries to start a conversation with Bob, she initiates the key exchange as shown in Fig. 2 when she sends the first message to Bob. Once Alice initiates the sending process, the server sends IKB, SPKB, SignB, and one OPKB if available. After receiving the public keys, she verifies the signature SignB with IKB. If the verification fails, she aborts the protocol. After successfully verifying SignB, she generates an ephemeral key pair (eka, EKA) and computes $\mathrm{DH} 1, \mathrm{DH} 2, \mathrm{DH} 3$ and $\mathrm{DH} 4$. If Bob ran out of OPKB and none was sent by the server, she will not compute $\mathrm{DH}$. After computing the three or four $\mathrm{DH}$, she enters the concatenation of all the $\mathrm{DH}$ into a KDF to generate the keys $c k$ and $m k$. mk is used to encrypt the message $M$ using an AEAD with additional data AD and ck is used in the ratcheting process. She then generates a ratcheting key pair (rcka, RCKA) and sends the cipher text $C, R C K A$ and EKA to the server which then forwards it to Bob. Once Bob is online, he receives EKA and $C$ and an indication of the OPKB that was sent to Alice.

Then, he computes DH1, DH2, DH3 and DH4. If an OPKB was not sent to Alice, he will not compute DH4 and will generate more (opk, OPK) pairs and send to server. After computing the three or four $\mathrm{DH}$, he also generates ck and mk using a KDF and $A D$ the way Alice did and use them to decrypt $C$ using the AEAD scheme. A security code will be created by hashing IKA and IKB and Alice and Bob can use it for authentication.

At this stage, a session has been created between Alice and Bob. Bob can now delete the (opk, OPK) pair used and Alice will delete the (eka, EKA) as they will not be used again. They can also delete the DH. Since both Alice and Bob register their public keys with the server at install time as shown in Fig. 1, Alice can get all the information required to send a message to Bob even if Bob is offline and when Bob gets online, he can read her message and send a reply even if she is offline. This is what makes the Trust Establishment asynchronous. 


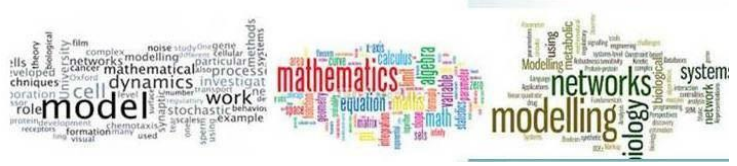

Journal, Advances in Mathematical \& computational Sciences

Vol. 8 No. 3 September, 2020

www.mathematics-computationaljournal.info

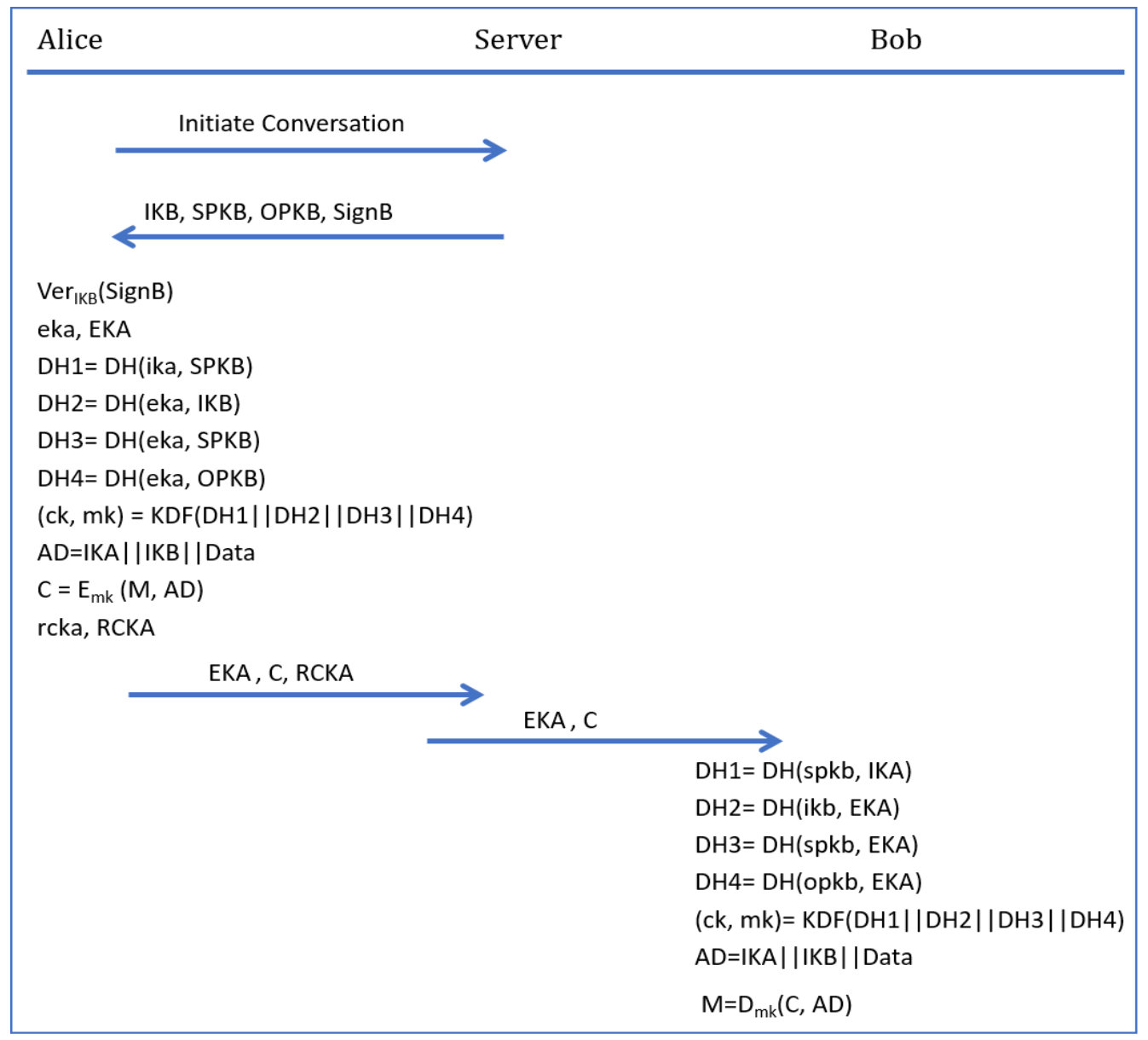

Figure 2: The Signal Key Exchange

\subsection{Messaging}

Once Alice and Bob complete the key exchange and have shared secret (ck0, mk0), they start a process called Double Ratcheting. The Double Ratcheting protocol specified in Signal documentation[14] has two ratcheting protocols; Asymmetric and Symmetric.

When sending the first message after key exchange, Alice also sends her first RCKA. When Bob wants to reply to Alice's message, he generates his (rckb, RCKB) pair and performs a DH with RCKA. He uses that DH and the shared secret rk0 to generate a new rk1 and ck1 using KDF2. The use of the DH to generate new keys is the Asymmetric ratchet step. The new ck1 is used to generate a new pair of secrets ck2 and mk1. This is the Symmetric ratchet step. Bob now uses the new secret $m 1$ to encrypt his reply and sends the cipher text together with RCKB. Alice follows the same procedure when she replies to Bob. This way, they both take turns in introducing a new DH into the protocol, hence achieving Backward Secrecy. Fig.3 gives further explanation of this process. 


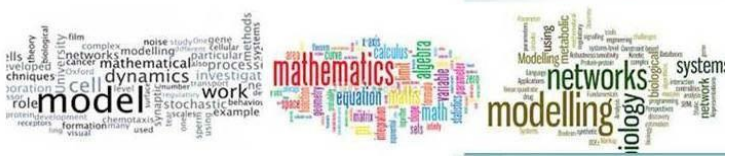

Journal, Advancesin Mathematical \& computational Sciences Vol. 8 No. 3 September, 2020

If Bob has not replied the message sent by Alice and she sends another message. Asymmetric ratchet cannot be applied since there is no new DH. In that case, she continues applying Symmetric ratchet for each message she sends until Bob replies and an Asymmetric ratchet is performed. Bob also does the same when sending consecutive messages. Fig. 4 shows the process of sending consecutive messages.

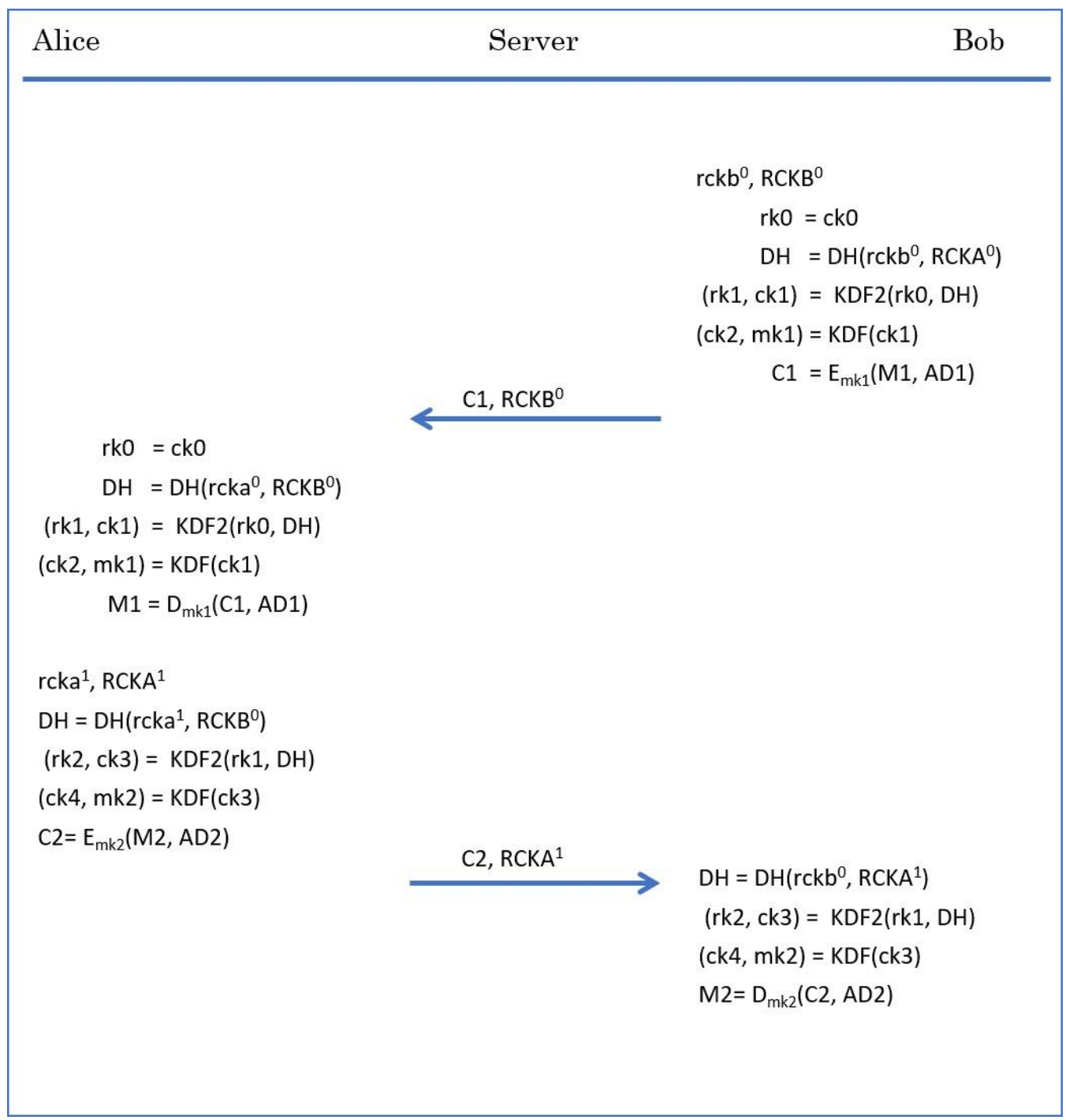

Figure 3: The Ratcheting Protocol: This shows what happens when Alice and Bob take turns in sending messages. 


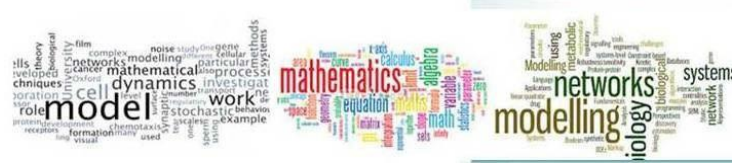

Journal, Advancesin Mathematical \& computational Sciences

Vol. 8 No. 3 September, 2020

www.mathematics-computationaljournal.info

Once a ck or mk is used, it can be deleted. The rk is only deleted when a new rk is generated from an asymmetric ratchet step. The secrets used by Alice in encryption form her sending chain while that of decryption is her receiving chain. Alice's sending chain is therefore equivalent to Bob's receiving chain and vice versa. A new sending and receiving chain is formed whenever an asymmetric ratchet step is performed. The message number in the sending chain is included in the message to track out of order messages.

\section{CRYPTOGRAPHIC PRIMITIVES}

The implementation of the Signal Protocol in Signal uses curve25519 Elliptic Curve Digital Signature Algorithm for signing and verifying messages. The hash function used is SHA-512. AES in CBC mode with HMAC-Sha256 authentication is used as AEAD and the key derivation functions are HMAC based. These cryptographic primitives are all considered to be secure and the Signal protocol has no known major vulnerabilities[9]. This work focuses on improving the efficiency of the Signal Protocol using some efficient state-of-the-art cryptographic primitives suggested in [3] to replace the existing primitives. The primitives considered are Blake2 hash function and Chacha20 with Poly1305 AEAD.

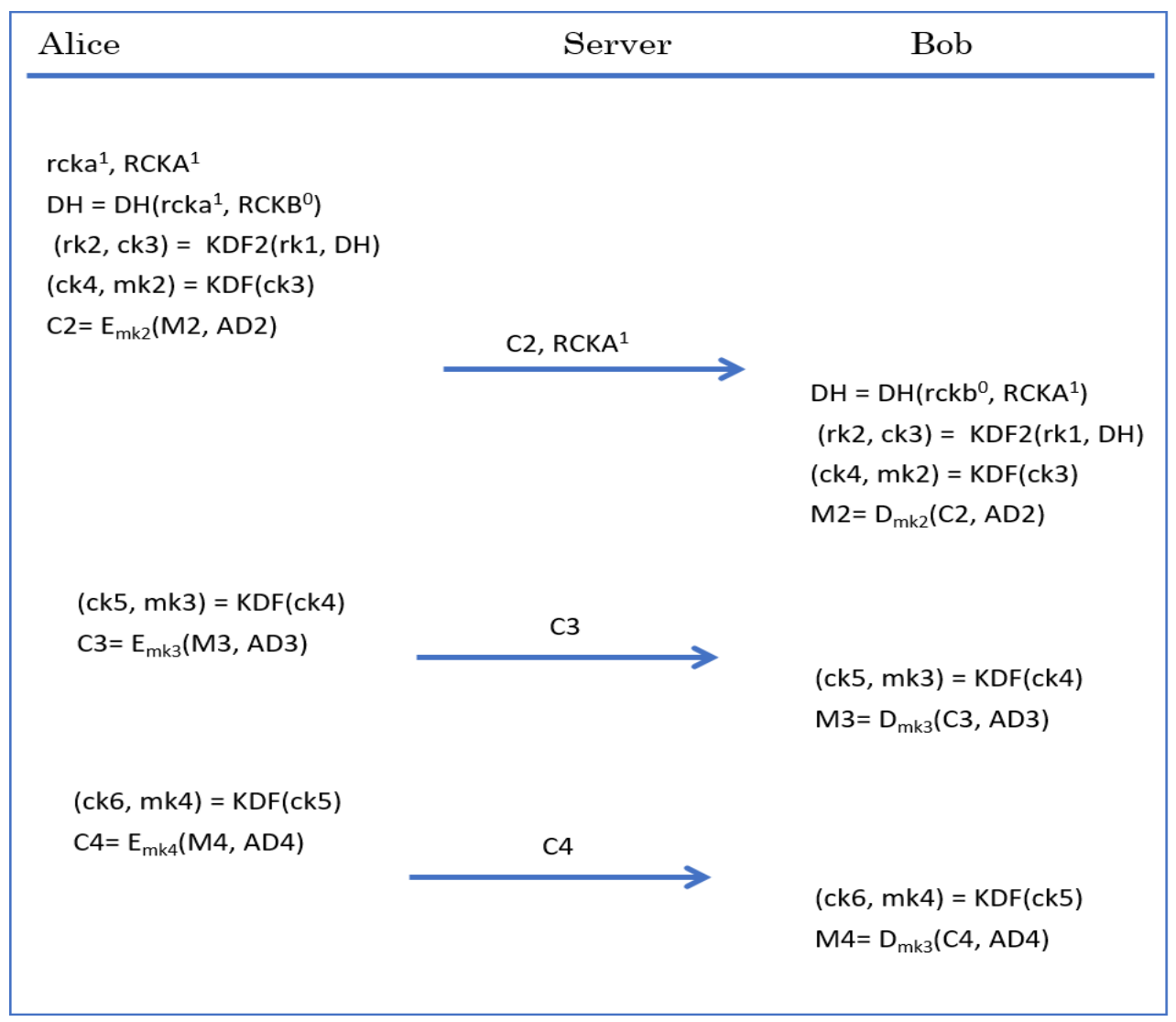

Figure 4: The Ratcheting Protocol: This shows what happens when Alice and Bob take turns in sending messages. 


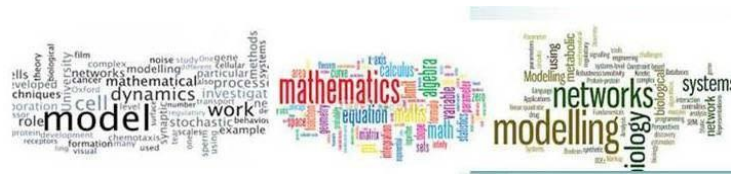

Journal, Advancesin Mathematical \& computational Sciences

Blake2 is a successor of Blake, which is one of the SHA-3 finalist [6]. It is a reliable hash function considered to be even faster than MD5 hash if properly implemented[6]. It is also resistant to different forms of attack[11][5]. ChaCha20 is a fast stream cypher which is sometimes used as a block cipher[7]. It uses Addition Rotation and Xor (ARX) which is more CPU friendly than the mixed columns and mixed rows used in AES[7]. Poly1305 is a fast Message Authentication Code [7]. It can be used together with Poly1305 in an AEAD. Chacha20/Poly1305 AEAD is already used in TLS[7]. It is also resistant to different attacks[12].

An application was created to test the running time of the proposed cryptographic primitives against the existing one. The application takes the inbuilt Java implementation of SHA-512 as used by Signal and measures the time it takes to take to hash a public key, a short text and a $1.4 \mathrm{MB}$ file. It then takes the Blake2b implementation and measures the time it will take to perform the same hash. The application also measures the running time of performing encryption and decryption of a short text and a 1.4 MB files using AES/CBC/HMAC-sha256 and Chacha20/Poly1305. The tests were performed ten times each and the average time was measured.

From the results, the average execution time of hashing keys with Blake2b was 67,880 ns while that of SHA-512 was $90,647 \mathrm{~ns}$ which is higher. The average execution time of Blake2b when hashing a short text was $59,156 \mathrm{~ns}$ and that of SHA-512 was $84,956 \mathrm{~ns}$ which is also higher. The average execution time of hashing a $1.4 \mathrm{MB}$ file using Blake2b was $8,019,587$ and it is also than that of SHA-512 which was 9,309,547ns (see table 1).

Table 1: Comparison of the efficiency of Blake2 and SHA-512

\begin{tabular}{|c|c|c|}
\hline & \multicolumn{2}{|c|}{ Time (ns) } \\
\hline & Blake2b & Sha512 \\
\hline Test 1 & 85,375 & 145,092 \\
\hline Test 2 & 57,383 & 106,369 \\
\hline 乞 Test 3 & 55,984 & 101,238 \\
\hline$\underline{v}$ Test 4 & 80,710 & 90,974 \\
\hline$\frac{0}{0}$ Test 5 & 55,051 & 61,115 \\
\hline J Test 6 & 61,583 & 62,982 \\
\hline$\underset{\tilde{\sigma}}{\frac{\tau}{\tilde{n}}}$ Test 7 & 55,984 & 63,449 \\
\hline I Test 8 & 85,841 & 101,703 \\
\hline Test 9 & 55,051 & 52,252 \\
\hline Test 10 & 85,842 & 121,298 \\
\hline Average: & 67,880 & 90,647 \\
\hline
\end{tabular}

\begin{tabular}{|c|c|c|c|}
\hline & \multicolumn{2}{|c|}{ Time (ns) } \\
\hline & & Blake2b & Sha512 \\
\hline \multirow{11}{*}{ 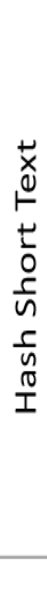 } & Test 1 & 57,384 & 95,173 \\
\hline & Test 2 & 56,451 & 79,777 \\
\hline & Test 3 & 57,850 & 86,309 \\
\hline & Test 4 & 65,315 & 84,909 \\
\hline & Test 5 & 56,450 & 92,840 \\
\hline & Test 6 & 55,983 & 93,773 \\
\hline & Test 7 & 56,450 & 87,241 \\
\hline & Test 8 & 55,517 & 66,714 \\
\hline & Test 9 & 56,450 & 72,312 \\
\hline & Test 10 & 73,712 & 90,507 \\
\hline & Average: & 59,156 & 84,956 \\
\hline
\end{tabular}

\begin{tabular}{|c|c|c|c|}
\hline & \multicolumn{2}{|c|}{ Time (ns) } \\
\hline & & Blake2b & Sha512 \\
\hline & Test 1 & $8,698,950$ & $9,703,860$ \\
\hline & Test 2 & $7,377,266$ & $8,418,098$ \\
\hline$\underline{0}$ & Test 3 & $9,318,504$ & $9,631,081$ \\
\hline iٓ & Test 4 & $7,842,398$ & $9,799,032$ \\
\hline$\sum_{+}^{\infty}$ & Test 5 & $8,308,930$ & $9,521,446$ \\
\hline i & Test 6 & $7,812,074$ & $9,544,306$ \\
\hline$\frac{r}{n}$ & Test 7 & $7,192,986$ & $8,149,842$ \\
\hline I & Test 8 & $7,815,806$ & $9,660,005$ \\
\hline & Test 9 & $8,064,934$ & $8,754,468$ \\
\hline & Test 10 & $7,764,021$ & $9,913,332$ \\
\hline & Average: & $8,019,587$ & $9,309,547$ \\
\hline
\end{tabular}




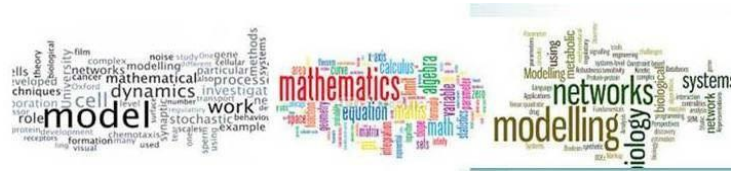

Journal, Advancesin Mathematical \& computational Sciences Vol. 8 No. 3 September, 2020

www.mathematics-computationaljournal.info

Table 2: Comparison of the efficiency of encrypting and decrypting a short text using AES/CBC/HMAC and Chacha20/Poly1303 AEAD schemes

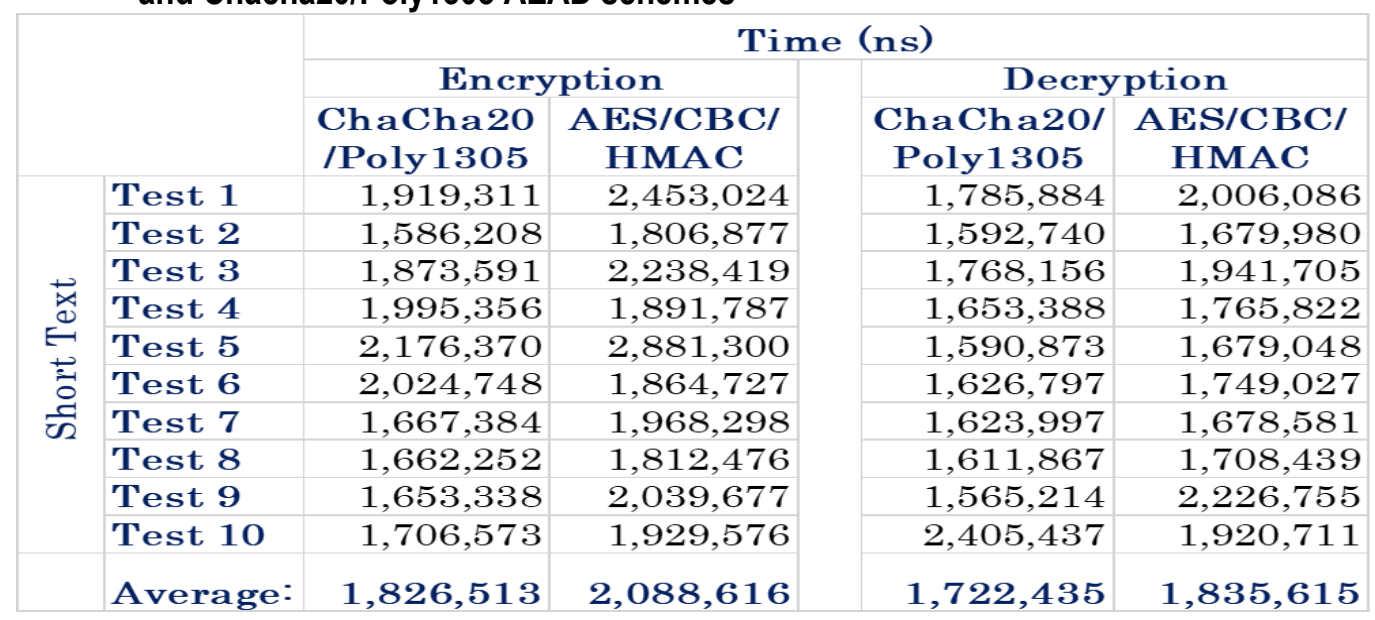

Table 3: Comparison of the efficiency of encrypting and decrypting a $1.4 \mathrm{MB}$ File using AES/CBC/HMAC and Chacha20/Poly1303 AEAD schemes

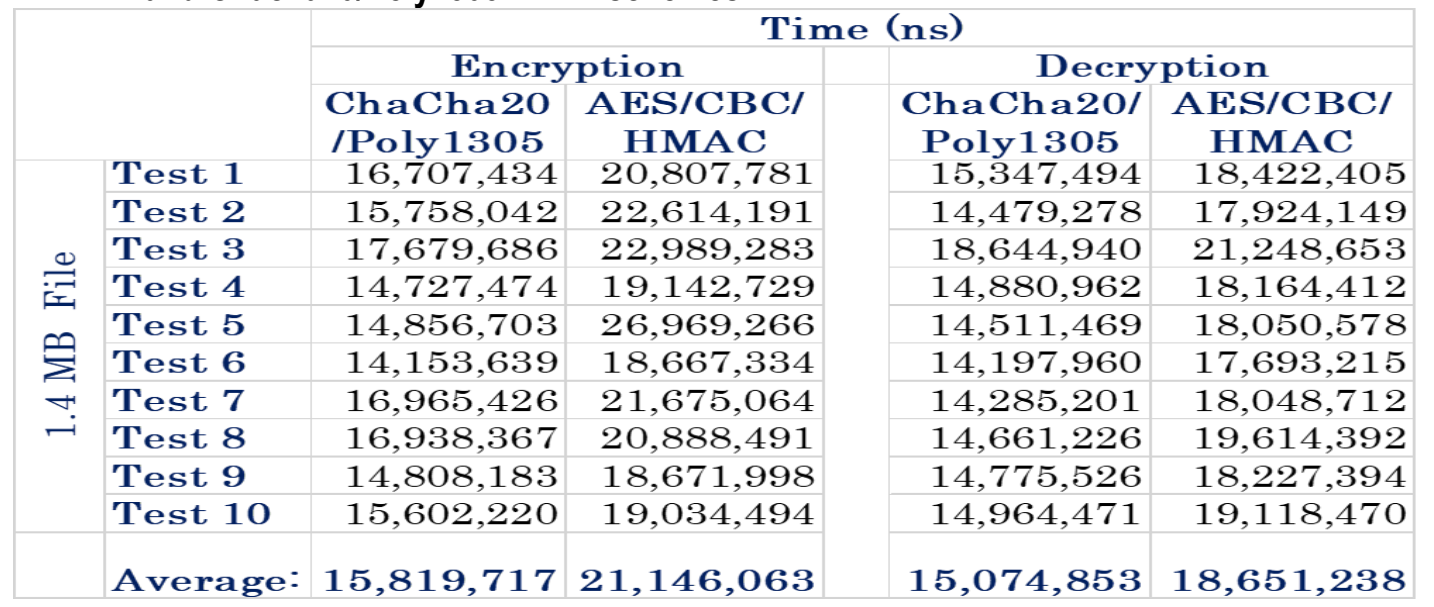

As shown in table 2, the average execution times for encrypting and decrypting a short text using Chacha20/Poly1305 were 1,826,513ns and 1,722,435ns respectively. Whereas, the average execution times for encrypting and decrypting same text using AES/CBC/HMAC-sha256 were 2,088,616ns and 1,835,615ns respectively. For $1.4 \mathrm{MB}$ file encryption and decryption, the average execution times for Chacha20/Poly1305 were $15,819,717 \mathrm{~ns}$ and that of AES/CBC/HMAC-sha256 were 18,651,238ns. Table 3 provides more information on the speed of file encryption and decryption. In both cases, both encryption and decryption times in Chacha20/Poly1305 were lower than the corresponding times in AES/CBC/HMAC-sha256. 


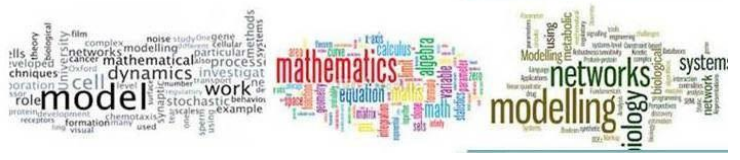

Journal, Advancesin Mathematical \& computational Sciences

Vol. 8 No. 3 September, 2020

www.mathematics-computationaljournal.info

\section{THE IMPROVED SIGNAL}

The cryptographic primitives were changed in the android version of the Signal application available in Open Whisper System's GitHub page[1]. The automatic tests in the Signal application was used to ensure that changes made to the code did not cause any logic errors. To further test the application, it was installed on another phone and messages were sent. The security code was also verified.

When the improved application was used to send a message to a user using the old application, the messages failed to deliver. This is expected because the key exchange will fail since the second user was using SHA-512 in $\operatorname{Sig}_{i k b}(S P K B)$ and the first user is using Blake2 in the $\operatorname{Ver}_{1 K B}(\operatorname{SignB})$. Figure 5 shows the result of sending messages with the improved application.

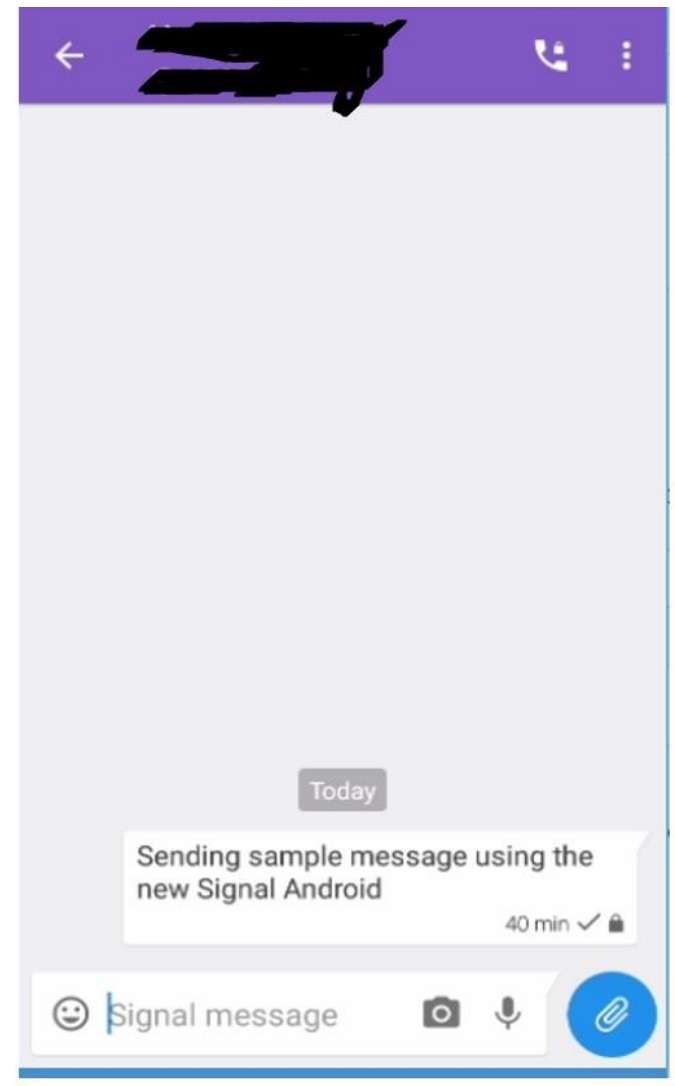

Message successfully sent using the new Signal Android

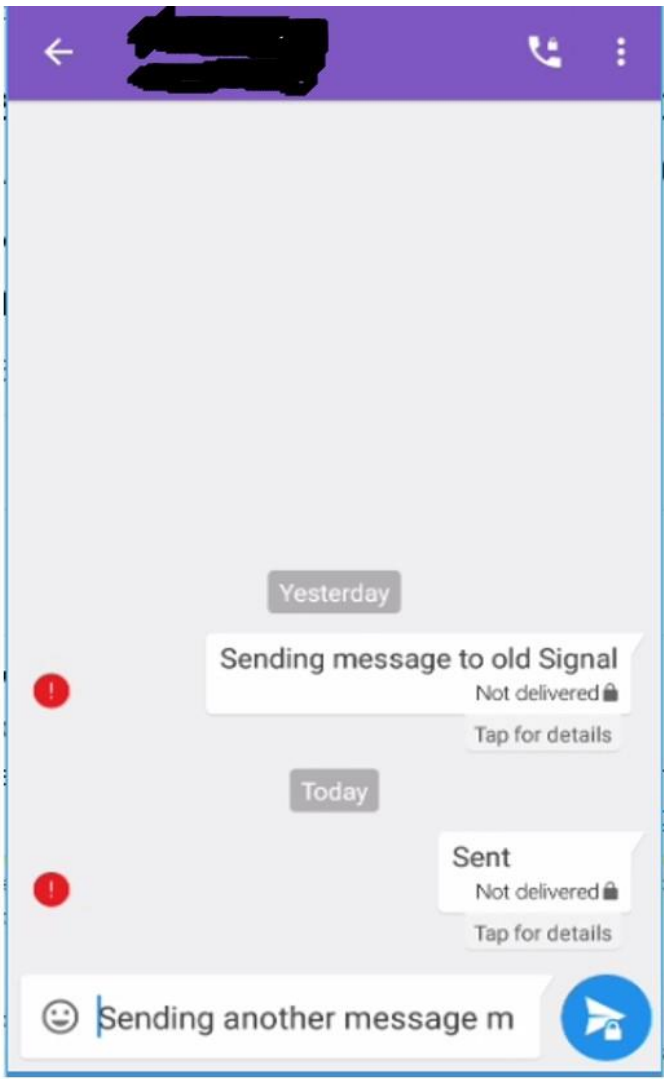

Message sent to original Signal android with the new Application failed to deliver

Figure 5: The result of sending messages using the new Signal Android. 


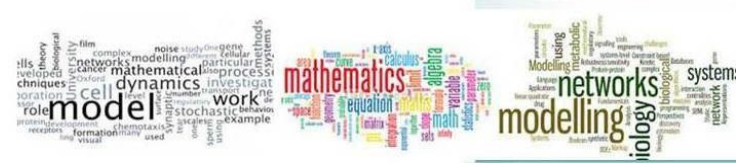

Journal, Advancesin Mathematical \& computational Sciences

Vol. 8 No. 3 September, 2020

www.mathematics-computationaljournal.info

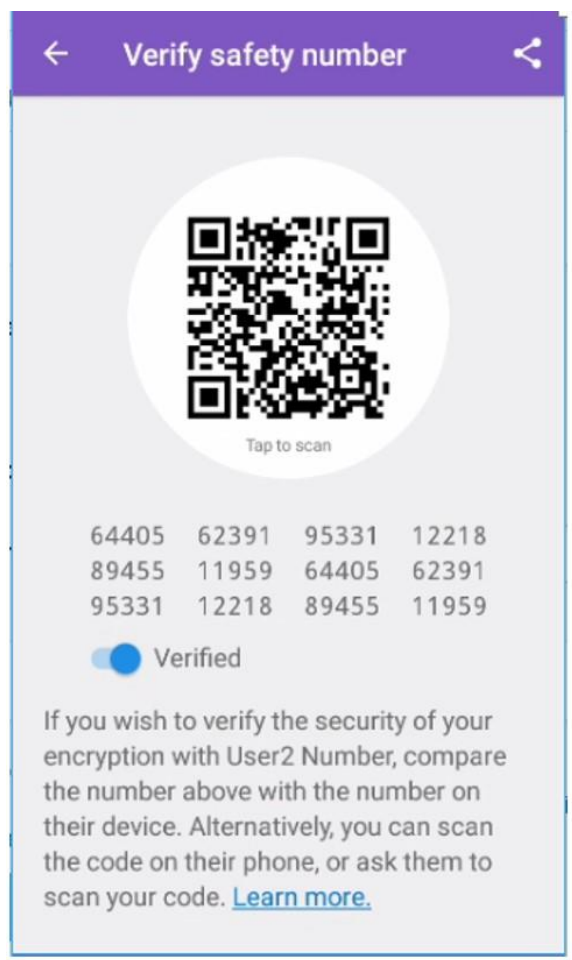

Security code successfully generated and verified using the new Application

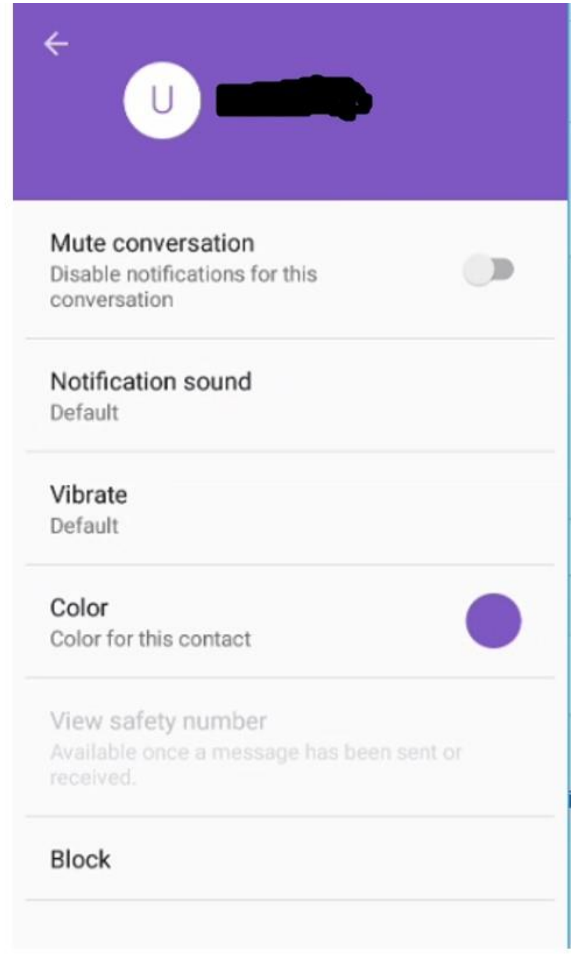

Security code cannot be generated because there is no successfully sent message from the new application to the original one

Figure 6: The result of verifying security code using the new Signal Android.

The verification of the security code between the two users using the improved application was also successful, but verification could not be performed with the user using the old application since the key exchange failed and the security code was not generated. Figure 6 shows the result of verifying security code using the improved application.

\section{CONCLUSION}

In this work, it was shown that the implementation of Blake2 hash function on short text and files is more efficient than that of sha512. Chacha20 with Poly1305 AEAD was also proved to be faster than AES in CBC mode with HMACSha256. Since the Signal Protocol is independent of any cryptographic algorithm or hash function, replacing the hash function and cryptographic algorithm did not require any alteration to the protocol itself. The cryptographic primitives can always be replaced with more secure ones if broken in the future. 


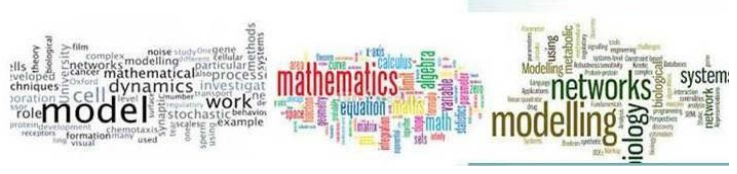

Journal, Advancesin Mathematical \& computational Sciences

Vol. 8 No. 3 September, 2020

www.mathematics-computationaljournal.info

\section{REFERENCES}

[1] Open whisper systems. https://github.com/WhisperSystems.

[2] Signal. https://signal.org/\#page-top. Accessed: 2018-10-06.

[3] Wireguard: Fast, modern, secure vpn tunnel. https://www.wireguard.com/.

[4] Jo"el Alwen, Sandro Coretti, and Yevgeniy Dodis. The double ratchet: Security notions, proofs, and modularization for the signal protocol. In Annual International Conference on the Theory and Applications of Cryptographic Techniques, pages 129-158. Springer, 2019.

[5] Jean-Philippe Aumasson, Willi Meier, Raphael C-W Phan, and Luca Henzen. Blake2. In The Hash Function BLAKE, pages 165-183. Springer, 2014.

[6] Jean-Philippe Aumasson, Samuel Neves, Zooko Wilcox-O'Hearn, and Christian Winnerlein. Blake2: simpler, smaller, fast as md5. In International Conference on Applied Cryptography and Network Security, pages 119-135. Springer, 2013.

[7] Daniel J Bernstein. Chacha, a variant of salsa20. In Workshop Record of SASC, volume 8, pages 3-5, 2008.

[8] Katriel Cohn-Gordon, Cas Cremers, Benjamin Dowling, Luke Garratt, and Douglas Stebila. A formal security analysis of the signal messaging protocol. In 2017 IEEE European Symposium on Security and Privacy (EuroS\&P), pages 451-466. IEEE, 2017.

[9] Katriel Cohn-Gordon, Cas Cremers, Benjamin Dowling, Luke Garratt, and Douglas Stebila. A formal security analysis of the signal messaging protocol. In Security and Privacy (EuroS\&P), 2017 IEEE European Symposium on, pages 451-466. IEEE, 2017.

[10] D I Dajoh, A U Atiku, and R S Naibi. Secure messaging: Analysis of signal protocol implementation in whatsapp and signal. Journal of Digital Inovations and Contemp Res. in Sc., Eng. and Tech., 6(3):63-72, 2018.

[11] Jian Guo, Pierre Karpman, Ivica Nikoli'c, Lei Wang, and Shuang Wu. Analysis of blake2. In Cryptographers' Track at the RSA Conference, pages 402-423. Springer, 2014.

[12] Kazuya Imamura, Kazuhiko Minematsu, and Tetsu Iwata. Integrity analysis of authenticated encryption based on stream ciphers. International Journal of Information Security, pages 1-19, 2016.

[13] Moxie Marlinspike and Trevor Perrin. The x3dh key agreement protocol. Open Whisper Systems Specifications: The X3DH Key Agreement Protocol, 2016.

[14] Trevor Perrin and Moxie Marlinspike. The double ratchet algorithm. GitHub wiki, 2016.

[15] Nik Unger, Sergej Dechand, Joseph Bonneau, Sascha Fahl, Henning Perl, lan Goldberg, and Matthew Smith. Sok: secure messaging. In Security and Privacy (SP), 2015 IEEE Symposium on, pages 232-249. IEEE, 2015. 